\title{
BMJ Open PEP-CoV protocol: a PEP flute-self-care randomised controlled trial to prevent respiratory deterioration and hospitalisation in early COVID-19
}

\author{
Annette Mollerup (D , ${ }^{1,2}$ Sofus Christian Larsen, ${ }^{1}$ Anita Selmer Bennetzen, ${ }^{1}$ \\ Marius Henriksen, ${ }^{3,4}$ Mette Kildevaeld Simonsen, ${ }^{5}$ Nina Weis (D) ,,6 \\ Linette Marie Kofod, ${ }^{7}$ Berit Lilienthal Heitmann (iD ${ }^{1,8}$
}

To cite: Mollerup A, Larsen SC, Bennetzen AS, et al. PEP-CoV protocol: a PEP flute-selfcare randomised controlled trial to prevent respiratory deterioration and hospitalisation in early COVID-19. BMJ Open 2021;11:e050582. doi:10.1136/ bmjopen-2021-050582

- Prepublication history for this paper is available online. To view these files, please visit the journal online (http://dx.doi org/10.1136/bmjopen-2021050582).

Received 01 March 2021 Accepted 27 May 2021

Check for updates

(C) Author(s) (or their employer(s)) 2021. Re-use permitted under CC BY-NC. No commercial re-use. See rights and permissions. Published by BMJ.

For numbered affiliations see end of article.

Correspondence to

Dr Annette Mollerup;

annette.mollerup@regionh.dk

\section{ABSTRACT}

Introduction Infection with SARS-CoV-2 may progress to severe pulmonary disease, COVID-19. Currently, patients admitted to hospital because of COVID-19 have better prognosis than during the first period of the pandemic due to improved treatment. However, the overall societal susceptibility of being infected makes it pivotal to prevent severe courses of disease to avoid high mortality rates and collapse of the healthcare systems. Positive expiratory pressure (PEP) self-care is used in chronic pulmonary disease and has been shown to prevent pneumonia in a high-risk cohort of patients with leukaemia. PEP flute self-care to prevent respiratory deterioration and hospitalisation in early COVID-19: a randomised trial (The PEP-CoV trial) examines the effectiveness on respiratory symptoms and need of hospital admission by regular PEP flute use among nonhospitalised individuals with confirmed SARS-CoV-2 infection and COVID-19 symptoms.

Methods and analysis In this randomised controlled trial, we hypothesise that daily PEP flute usage as add-on to usual care is superior to usual care as regards symptom severity measured by the COPD Assessment Test (CAT) at 30-day follow-up (primary outcome) and hospital admission through register data (secondary outcome). We expect to recruit 400 individuals for the trial. Participants in the intervention group receive a kit of 2 PEP flutes and adequate resistances and access to instruction videos. A telephone hotline offers possible contact to a nurse. The eight-item CAT score measures cough, phlegm, chest tightness, dyspnoea, activities of daily living at home, feeling safe at home despite symptoms, sleep quality and vigour. The CAT score is measured daily in both intervention and control arms by surveys prompted through text messages.

Ethics and dissemination The study was registered prospectively at www.clinicaltrials.gov on 27 August 2020 (NCT04530435). Ethical approval was granted by the local health research ethics committee (Journal number: H-20035929) on 23 July 2020. Enrolment of participants began on 6 October 2020. Results will be published in scientific journals.

Trial registration number NCT04530435; Pre-results.
Strengths and limitations of this study

- Using a randomised design, this study addresses an important evidence gap in the SARS-CoV-2 pandemic; how to mobilise the individual's self-care to prevent respiratory deterioration in COVID-19 with the use of a simple, cheap and accessible intervention, thus potentially avoid hospitalisation.

- This study is a niche project between a public health intervention and disease prevention in a clinical setting which may challenge a warranted non-selective recruitment as recruitment awaits the initiative from eligible participants.

- Due to the type of intervention, blinding of the participants and treatment providers is not feasible.

- COVID-19 is a novel disease and this study is explorative when using self-reported measurements from COPD treatment, that is, the COPD Assessment Test score as an outcome variable. In the absence of objectively measured values like oxygen saturation or body temperature as outcome variables, this calls for attention when discussing the results of the trial.

- There is a risk of contamination across arms as participants can acquire the positive expiratory pressure (PEP)-flute as over-the-counter medical equipment.

\section{INTRODUCTION}

The pandemic infection with SARS-CoV-2 may result in non-specific symptoms like fever, fatigue and dyspnoea, or it may progress to severe pulmonary disease, COVID-19. In Mid-January 2021, as reported by WHO, the number of people dying worldwide because of COVID-19 exceeded two million. Over time, we learn more about this new disease, for example, reports of a median time from symptom onset to development of pneumonia of approximately 5 days. ${ }^{1}$ COVID-19 seems to damage the respiratory system due to an over-reaction of the immune system with individual risk profiles of age and comorbidity. ${ }^{2}$ 
This may lead to acute respiratory distress syndrome, and in these cases, the median time from symptom onset to severe hypoxaemia and intensive care unit (ICU) admission has been reported to be approximately $7-12$ days. ${ }^{1}$ At a median follow-up period of 79 days among ICU patients, mortality was reported to be $37 \%$ in a Danish nationwide study. Hence, the COVID-19 disease burdens the healthcare systems even in countries without any restrictions as to ICU admission in times of a pandemic. ${ }^{3}$

At present, the disease trajectory is not easy to predict, ${ }^{1}$ and little is known of any measures or medication to alter the course of early-stage disease, that is, to prevent the need for hospitalisation and critical care. The PEP-CoV trial will investigate the effect of positive expiratory pressure (PEP) flute self-care on respiratory deterioration and hospital admission among non-hospitalised individuals with COVID-19 symptoms. If PEP flute self-care proves to be effective, it will be easy to implement as a public health intervention also in a global context. In the trial, participants have confirmed SARS-CoV-2 infection by positive PCR swab test and COVID-19 symptoms at study entry, hence, although no medical examination has been conducted, they are considered to be COVID-19 cases according to WHO COVID-19 case definitions. ${ }^{4}$

\section{Background and rationale}

Recent evidence suggests a poor prognosis whenever the COVID-19 disease has become so severe that hospital admission is needed. A large observational cohort study from UK found that within a minimal follow-up time of 2 weeks, $26 \%$ of patients admitted to acute care hospitals had died. ${ }^{5}$ Among patients in need of critical care facilities and/or receiving mechanical ventilation, the proportion of fatal outcome was $32 \%$ and $37 \%$, respectively. In the pandemic waves, healthcare systems face an imminent threat of collapse because of an overload of COVID-19 cases. The prognosis of having a severe course of disease due to COVID-19 is better now than in the first period of the pandemic because of improvements in treatment. Antiviral treatment with remdesivir, and dexamethasone, appears to have moderate effects. ${ }^{67}$ However, both treatments are administered only in cases when the patient is hospitalised and in need of oxygen. In the overall population, all are at risk of being infected, and this overall societal susceptibility makes prevention of severe courses of disease pivotal to the healthcare system.

A variety of symptoms have been observed in patients with COVID-19. The study by Docherty et al refers to clusters of symptoms on admission, that is, musculoskeletal symptoms (myalgia, joint pain, headache and fatigue); enteric symptoms (abdominal pain, vomiting and diarrhoea); and a mucocutaneous cluster. ${ }^{5}$ However, the most common symptom cluster involves respiratory symptoms, that is, cough, sputum and shortness of breath, accompanied by fever. When critically ill, the intensive care treatment includes mechanical ventilation with high oxygenation and positive end expiratory pressure (PEEP). PEEP increases functional residual capacity
(FRC) and reduces the work of breathing. The use of PEEP has been highlighted as a very important measure to avoid a critical course administered as continuous positive airway pressure (CPAP) by face masks or by use of a helmet. ${ }^{89}$ However, this treatment is for hospitalised patients. PEP is used as self-care in chronic inflammatory pulmonary diseases like chronic obstructive pulmonary disease (COPD) despite the lack of robust evidence. In a randomised controlled trial (RCT), PEP therapy as add-on to usual medical care had only minor effects among inpatients with acute exacerbation of COPD. The intervention led to more rapidly improved dyspnoea following discharge but had no impact on subsequent exacerbations and hospitalisations. ${ }^{10}$ Little is known of the potential effects of PEP as self-care in pneumonia prevention. Among patients with leukaemia, PEP alongside daily spirometry was superior to daily spirometry to prevent pneumonia (first pneumonia incidence per 1000 days, 2.17 vs $6.52, p=0.021$, intervention group and control group, respectively). ${ }^{11}$ The mechanically supported inflation of the alveoli and loosening of secretions by PEP presumably prevented manifest lung infiltrates without any adverse events (AEs). ${ }^{11}$ Among several effects, use of PEP can increase FRC and tidal volume, decrease hyperinflation and improve airway clearance. ${ }^{12}$ Moreover, in both healthy subjects and patients undergoing surgery, increased gas exchange and decreased atelectasis have been reported after PEP usage. ${ }^{12}$ Analogously, PEP may have beneficial effects on the progression of symptoms in the COVID-19 trajectory. Airway clearance techniques (ACTs) appear to be safe, and the PEP flute has been shown as effective as other ACTs. ${ }^{13}$

Most current research on SARS-CoV-2 and COVID-19 relates to screening measures, vaccine development and optimising hospital treatment, that is, the bottom and top ends of a pyramid, which depict the relationship between populational size, setting and treatment options (figure 1). It is likely that we have this pandemic for several years until we have reached a high level of immunity in the population either by natural spread of the disease or via an efficient vaccination programme, and measures are needed to help the SARS-CoV-2-infected individual at home to overcome the course of disease with less symptoms and strain. Based on the hypothesis that the regular use of a PEP flute may prevent the progression of respiratory symptoms in non-hospitalised individuals with SARS-CoV-2 infection, a PEP flute intervention, feasible for home use, may prevent prolonged disease courses, long-term sequelae and costly hospital admissions.

\section{Study objectives and hypotheses}

The aim of the present study was to explore the effectiveness on respiratory symptoms by regular use of PEP among SARS-CoV-2-infected, non-hospitalised individuals with COVID-19 symptoms. The primary objective was to examine the effect of PEP flute use on self-reported symptoms during 30 days of follow-up. We hypothesise that PEP flute use has positive effects on self-reported 


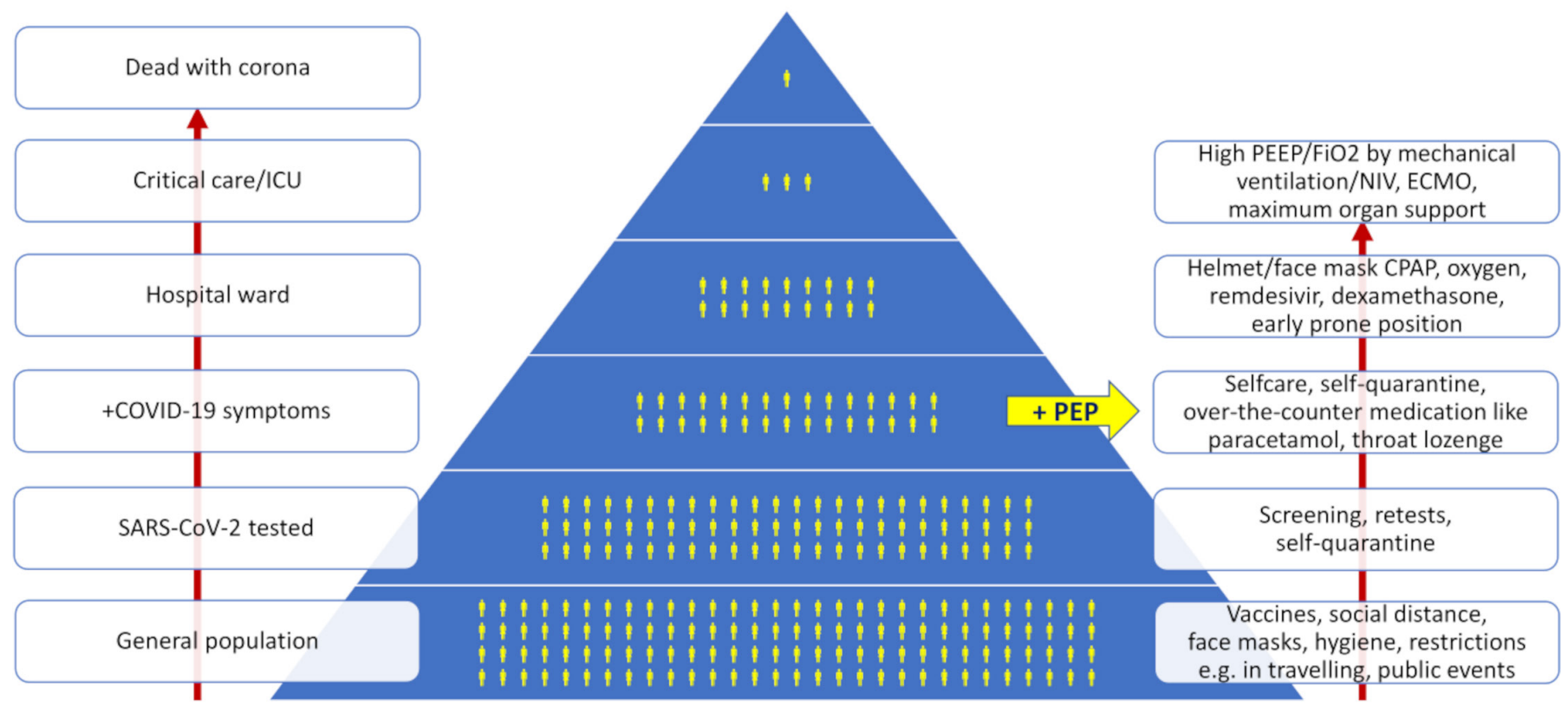

Figure 1 Relationship between population/setting and level of care/treatment options of SARS-CoV-2 and COVID-19. Upwards arrows indicate disease trajectory and higher level of care accordingly (to the left) and add-ons of treatment options (right side). CPAP, continuous positive airway pressure; ECMO, extracorporeal membrane oxygenation; $\mathrm{FiO}_{2}$, inspired oxygen fraction; ICU, intensive care unit; NIV, non-invasive ventilation; PEEP, positive end expiratory pressure; PEP, positive expiratory pressure.

respiratory symptoms such as dyspnoea, coughing and perceived mucus clearance through beneficial effects on lung function and airway clearance. Second, we expect a lower rate of hospitalisation and use of antibiotics in the intervention group as compared with the usual care group, the latter in case of a bacterial superinfection.

\section{METHODS}

\section{Trial design and setting}

The PEP-CoV trial is designed as a randomised, controlled, open-label trial with two parallel groups and consecutive inclusion. The trial is investigator-initiated and hosted by the Parker Institute, a part of Copenhagen University Hospital Bispebjerg-Frederiksberg. The participants are recruited from the Capital Region and Region Zealand in Denmark (in total, approximately 2.7 million citizens). The trial registration data set is displayed in table 1 .

\section{Patient and public involvement}

Ideation of the trial intervention was based on anecdotal evidence of a PEP flute's beneficial effects in a single case of COVID-19. Personal communication with COVID-19 convalescents has contributed to the designing process of the study. However, due to the ongoing pandemic crisis, further patient and public involvement in the research process has not been feasible.

The study duration is 6 months and the primary endpoint is COPD Assessment Test (CAT) score after 30 days of active intervention. Follow-ups of CAT scores are also scheduled at 90 and 180 days postbaseline. The study's enrolment, intervention and assessment schedules according to Standard Protocol Items: Recommendations for Interventional Trials (SPIRIT) Guidelines are outlined in table $2 .{ }^{14}$

\section{Trial population and eligibility criteria}

To avoid unnecessary spread of the SARS-CoV-2, any contact, that is, oral information, consent and screening, is provided over phone and by use of secured electronic communication via the public 'Digital Post' system (electronic mailbox for letters from Danish authorities) administered by the platform 'e-Boks' (https://www. e-boks.com/danmark/en). This system is linked to the individual's personal identification number-a national identification number which is part of the personal information stored in the Danish Civil Registration System. Daily information of positive results from the SARS-CoV-2 PCR tests is provided from the Departments of Microbiology at Copenhagen University Hospitals Rigshospitalet, Hvidovre Hospital and Herlev Hospital, which covers the overall Capital Region, and the Department of Microbiology, Slagelse Hospital, covering the entire Region Zealand. Based on these data, individuals eligible for study participation receive study information and invitation electronically via e-Boks. The individual may then contact the project directly via e-mail or phone or leave a phone number for a subsequent call from the study staff (AM and ASB).

The inclusion/exclusion criteria for the trial are described in table 1 . The exclusion criteria are deliberately 


\begin{tabular}{ll} 
Table 1 WHO trial registration data set \\
\hline Data category $\quad$ Information \\
\hline $\begin{array}{l}\text { Primary registry } \\
\text { and trial identifying } \\
\text { number }\end{array}$
\end{tabular}

Date of registration in 27 August 2020

primary registry

\begin{tabular}{|c|c|}
\hline $\begin{array}{l}\text { Secondary identifying } \\
\text { numbers }\end{array}$ & $\begin{array}{l}\text { Danish Data Protection Agency (P-2020-879) } \\
\text { Health Research Ethics (H-20035929) }\end{array}$ \\
\hline $\begin{array}{l}\text { Sources of monetary } \\
\text { or material support }\end{array}$ & $\begin{array}{l}\text { Innovation Fund Denmark (0211-00023B) and the } \\
\text { Danish Nursing Council (grant number: } \mathrm{n} / \mathrm{a})\end{array}$ \\
\hline Sponsor & The Parker Institute, Copenhagen, Denmark \\
\hline $\begin{array}{l}\text { Contact for public } \\
\text { queries }\end{array}$ & $\begin{array}{l}\text { Annette Mollerup, PhD (annette.mollerup@regionh.dk) } \\
\text { The Parker Institute } \\
\text { Copenhagen University Hospital Bispebjerg- } \\
\text { Frederiksberg } \\
\text { Ndr. Fasanvej 57, } 2000 \text { Frederiksberg, Denmark } \\
\text { +45 } 38163102\end{array}$ \\
\hline $\begin{array}{l}\text { Contact for scientific } \\
\text { queries }\end{array}$ & Annette Mollerup, PhD (annette.mollerup@regionh.dk) \\
\hline Public title & $\begin{array}{l}\text { COVID-19: symptoms and respiratory self-care } \\
\text { (in Danish: COVID-19 sygdom: symptomer og } \\
\text { vejrtrækningsøvelser) }\end{array}$ \\
\hline Scientific title & $\begin{array}{l}\text { PEP flute self-care to prevent respiratory deterioration } \\
\text { and hospitalisation in early Covid-19: a randomised } \\
\text { trial (acronym: The PEP-CoV trial) }\end{array}$ \\
\hline $\begin{array}{l}\text { Countries of } \\
\text { recruitment }\end{array}$ & Denmark \\
\hline Health condition(s) & $\begin{array}{l}\text { Adults aged } 18 \text { or older with a positive SARS-CoV-2 } \\
\text { test and symptoms of COVID- } 19\end{array}$ \\
\hline Intervention & $\begin{array}{l}\text { Active comparator: daily use of PEP flute and daily } \\
\text { self-monitoring of symptoms for } 30 \text { days as add-on to } \\
\text { usual care } \\
\text { Comparator: daily self-monitoring of symptoms and } \\
\text { usual care including self-care recommended by the } \\
\text { Danish Health Authorities (self-quarantine, sufficient } \\
\text { intake of liquid especially in case of high body } \\
\text { temperature, over-the-counter symptom relieving } \\
\text { medication) }\end{array}$ \\
\hline
\end{tabular}

\begin{tabular}{|c|c|}
\hline $\begin{array}{l}\text { Key inclusion and } \\
\text { exclusion criteria }\end{array}$ & $\begin{array}{l}\text { Inclusion criteria: } \\
\text { 1. Aged 18years or older. } \\
\text { 2. Positive SARS-CoV test. } \\
\text { 3. Symptoms of COVID-19 according to the } \\
\text { COVIDmeter; at least one respiratory symptom } \\
\text { (cough, sneezing, shortness of breath, chest pain } \\
\text { or runny nose) and one general symptom. } \\
\text { 4. Access to use a smartphone. } \\
\text { 5. Can reply to a questionnaire in Danish (sent on } \\
\text { email, text-message or via telephone interview) as } \\
\text { assessed by the investigator. } \\
\text { 6. Given informed consent. } \\
\text { Exclusion criteria: } \\
\text { 1. Any condition or impairment that, in the opinion } \\
\text { of the investigator, makes a potential participant } \\
\text { unsuitable for participation or which obstruct } \\
\text { participation, such as psychiatric disorders, } \\
\text { individuals, habitually using a PEP flute, } \\
\text { participation in other clinical COVID trials or } \\
\text { persons living in the same household as existing } \\
\text { participants in the trial. } \\
\text { 2. Hospitalised patients or nursing home residents. }\end{array}$ \\
\hline Study type & $\begin{array}{l}\text { Interventional, open-label trial with randomisation to } \\
\text { two parallel groups } \\
\text { Primary purpose: prevention of respiratory } \\
\text { deterioration of symptoms and hospitalisation }\end{array}$ \\
\hline $\begin{array}{l}\text { Data of first } \\
\text { enrolment }\end{array}$ & October 2020 \\
\hline Target sample size & 400 \\
\hline Recruitment status & Recruiting \\
\hline Primary outcome & Day 30 CAT score (modified for the present study) \\
\hline
\end{tabular}

Continued
Table 1 Continued

\begin{tabular}{ll}
\hline Data category & Information \\
\hline Key secondary & 1. Hospital admissions on days 30,90 and 180. \\
outcomes & 2. Use of antibiotics in case of superinfection. \\
& 3. COVID-19 symptoms, days 30,90 and 180. \\
& 4. CAT score, days 90 and 180. \\
& 5. Number of participants with serious adverse \\
& 6. Conts during the 30 -day intervention period. \\
\hline
\end{tabular}

COVIDmeter, the Danish Health Authority surveillance of symptoms reported by the public to a designated website; CAT, COPD Assessment Test consisting of eight items on a scale from 0 to 5: cough, phlegm, chest tightness, dyspnoea, activities of daily living at home, feeling safe at home despite symptoms (because of actual selfquarantine, modified for the present study from feeling safe at leaving home despite symptoms), sleep quality and vigour.

CAT, COPD Assessment Test; PEP, positive expiratory pressure.

few to reflect the target population and to promote a highly scalable public health implementation given a successful intervention. Various symptoms have been associated with COVID-19, ${ }^{515}$ and early Danish reports indicated that the most frequent self-reported respiratory symptoms in COVID-19 $(\mathrm{n}=308)$ were cough $(71 \%)$ and shortness of breath $(54 \%) .{ }^{16}$ However, recent findings have suggested that approximately three quarters of people with SARS-CoV-2 infection are asymptomatic on the day of the test. ${ }^{17}$ As the rationale for the potential effect of a PEP flute in a course of SARS-CoV-2 infection and COVID-19 involves the progression of respiratory symptoms, at least one reported respiratory symptom is required at enrolment. A screening manual has been developed and questions of symptoms according to the COVIDmeter ${ }^{18}$ are posed after given consent (table 2).

\section{Randomisation and blinding}

The participants reply to a telephone-administered baseline questionnaire before randomisation. Subsequently, the participant is randomly allocated to a treatment or control arm using an appropriate statistical software embedded in REDCap, an online web-based clinical trial management application (Vanderbilt University, Nashville, Tennessee, USA). ${ }^{19}$ The computer-generated random allocation is then unknown to the investigator and data collectors. As mortality prognosis to COVID-19 is higher in men and the elderly, ${ }^{5}$ the allocation is based on permuted random blocks and 1:1 stratified for the conditions sex and age ( $<60$ and $\geq 60$ years). Sex is determined through the unique Danish personal identification number as a binary variable.

As this is an 'open-label' trial, neither the health professionals delivering the interventions nor the participants are blinded to treatment allocation. Statistical analyses will be conducted blinded to the intervention group.

\section{Trial intervention}

The trial intervention is the regular use of a PEP flute in combination with usual care. A set of two PEP flutes and three airway resistances (equivalent to a resistance of $10-20 \mathrm{~cm} \mathrm{H}_{2} \mathrm{O}$ ) is delivered to the participants who are advised to use the PEP flute at least three times 
Table 2 Schedule for study enrolment, intervention and assessments

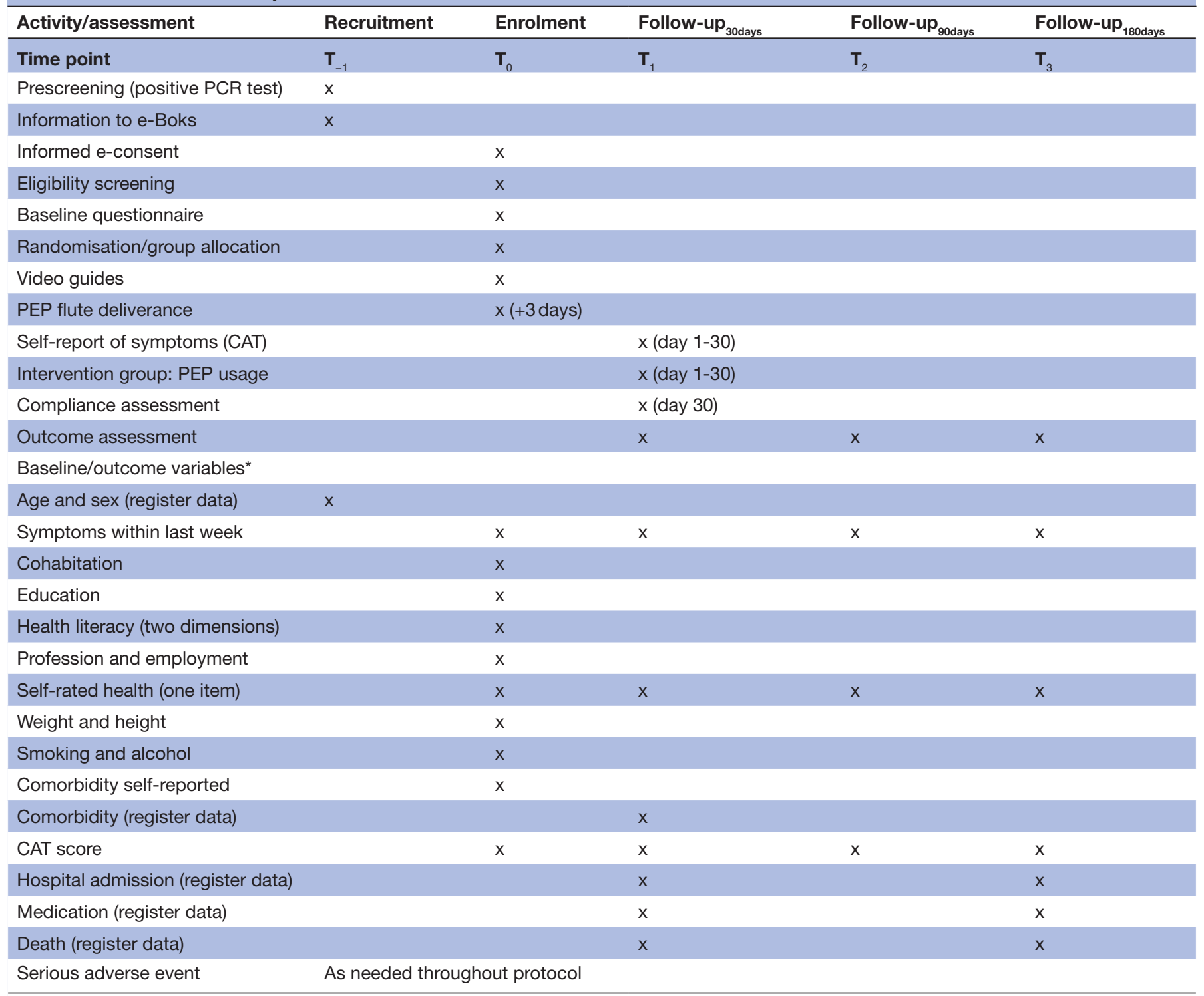

${ }^{*}$ All baseline and outcome variables are collected as questionnaire data unless stated otherwise.

CAT, COPD Assessment Test; PEP, positive expiratory pressure.

daily. Ideally, each session consists of $10-15$ breaths (for approximately $1 \mathrm{~min}$ ) performed twice with the participant sitting at an upright position. Two video guides (figure 2) are sent to the participant by e-Boks: one with instructions as to the rationale and how to use the PEP flute, including how to choose the suitable resistance; the other with instruction of hygienic maintenance, advised to be performed daily because of a manifest SARS-CoV-2 infection.

The use of a PEP flute is considered safe for even the weakest patient with lung disease..$^{20}$ The participants are instructed in using the flute with a pressure of approximately $10 \mathrm{~cm} \mathrm{H}_{2} \mathrm{O}$. If a person blows with full power, they might reach a pressure of approximately $50 \mathrm{cmH}_{2} \mathrm{O}$, whereas coughing generates a pressure in the lungs of $80-120 \mathrm{~cm} \mathrm{H} \mathrm{H}_{2} \mathrm{O} .{ }^{21}$ The participants will be advised to stop the PEP flute session in case of any discomfort.
Even among patients acutely ill with leukaemia and having neutropenia, no AEs were detected related to PEP usage. ${ }^{11}$ Despite this, the participants in the intervention group are encouraged to inform the project manager in case of any $\mathrm{AE}$ during the trial via the designated hotline or by e-mail.

Participants are advised to continue use of PEP in the active intervention period of 30 days or at least for as long as they still have respiratory symptoms. They receive daily text messages administered as an automated service by Twilio to prompt their reporting of CAT scores by links to a questionnaire in REDCap. Also, they are asked to report their present choice of airway resistance as well as the number of PEP flute sessions the previous day. These daily self-reports constitute assessment of treatment adherence. 
A
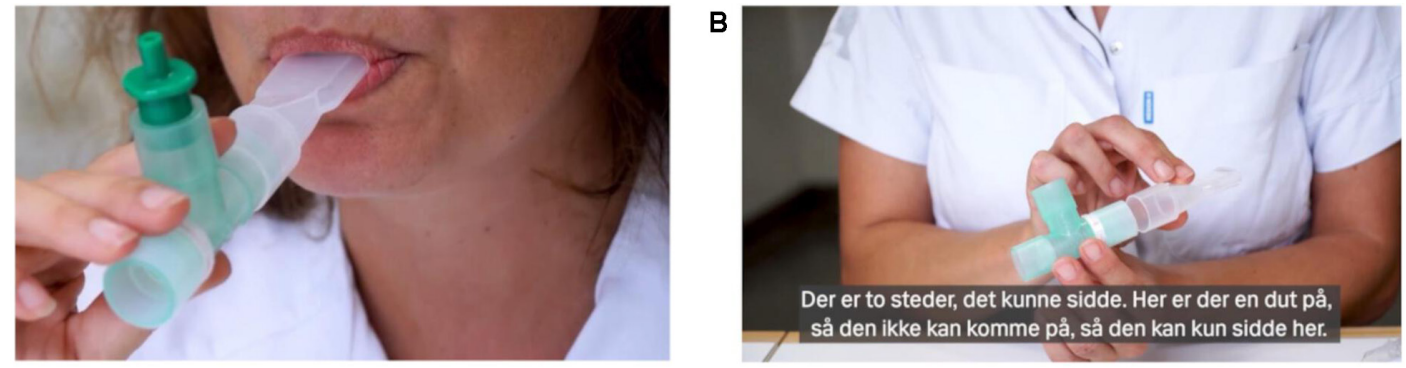

c

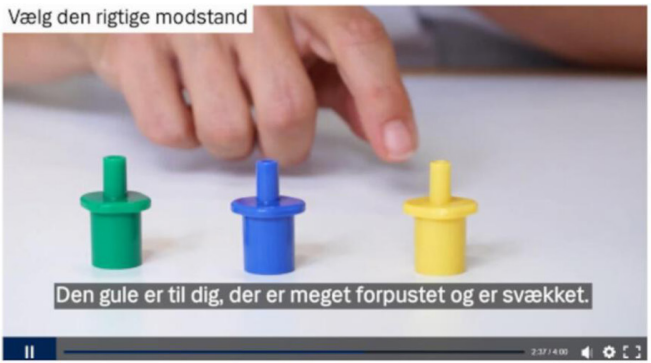

D

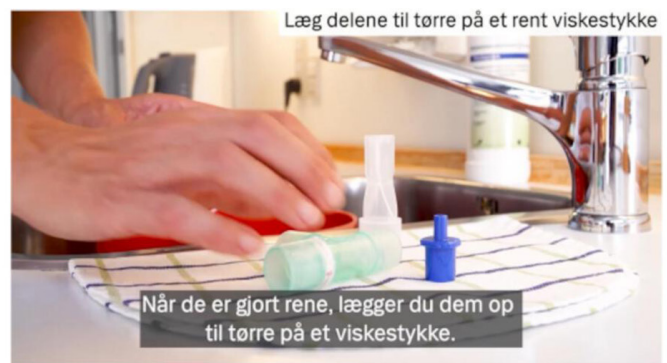

Figure 2 Still photos from instruction videos about PEP flute usage and hygienic maintenance. In detail, (A) how to use the PEP flute, (B) how to assemble the three parts of the flute correctly, (C) how to choose the suitable resistance and (D) how to perform hygienic maintenance of the PEP flute. Both videos including the shown subtitles in Danish are produced by the Communication Unit at Copenhagen University Hospital Hvidovre. PEP, positive expiratory pressure.

\section{Usual care}

As self-care in COVID-19, the Danish Health Authorities recommend sufficient intake of liquid especially in case of high body temperature; potential use of paracetamol when having myalgia, headache and fever; and a throat lozenge in case of sore throat. Otherwise, the citizen with a positive SARS-CoV-2 test is requested to perform selfquarantine and to pay special attention to hygiene and cleaning maintenance. The participants in the usual care group also receive daily text- messages to prompt their reporting of CAT scores by links to the electronic questionnaire.

To avoid attrition of the trial due to early recovery of symptoms, the project manager will contact the participants in both allocation groups by phone or text message approximately on day 15 to ask about their health condition and to answer to any potential concerns of continued participation in the trial. As part of the trial information, the participants in both groups are advised to contact their general practitioner, the COVID-19-specific clinics or the emergency medical services, if needed, as they would otherwise do if not participating in the trial.

\section{Measurements}

Data are collected both through questionnaires (primary outcome) and as register data (see table 2). With consideration of the participants' possibly affection with sickness at inclusion point, the questionnaire at baseline is deliberately delimited. The participants are asked about educational/professional background as healthcare professionals have both higher incidence of COVID-19 and are presumably better qualified to conduct disease self-care than lay persons. Health literacy will be measured by two dimensions, 'understanding' and 'engagement' (five items each), derived from the multidimensional Health Literacy Questionnaire and validated in a Danish general population survey. ${ }^{22}$ The understanding dimension covers 'understanding health information well enough to know what to do', whereas engagement covers 'the ability to actively engage with healthcare providers'. ${ }^{22}$ In addition to the aforementioned topics, the participants are asked a single item of self-rated health (on a 5-point Likert scale) and a few questions about smoking and alcohol habits.

\section{Primary outcome}

In the design phase of the trial, a valid COVID-19 symptom severity scale for outpatients was lacking, and emphasis was on the feasibility of the individual self-reporting symptoms while being sick with COVID-19. The CAT is a validated questionnaire designed to evaluate symptoms in patients with COPD. ${ }^{23}$ The CAT is free of use by curtesy of GlaxoSmithKline and is widely used both as a telemonitoring tool and to stratify the patients into groups based on the severity of symptoms. Even among the patients in the most unstable phase of COPD, daily selfreport of CAT is considered quick and easy for patients to use. ${ }^{24}$ The latter is important to ensure adequate data collection among the participants in the present trial. The eight items in the scale cover symptoms of cough, phlegm, chest tightness, dyspnoea, activities of daily living at home, feeling safe at home despite symptoms (modified for the present study from feeling safe at leaving home despite symptoms), sleep quality and vigour. The eight items sum up to a range of $0-40$ with higher scores indicating more symptom impairment. Although not validated for COVID-19 trials, the CAT scale is considered useful in the present study because several similar items 
(dyspnoea, cough, fatigue, sputum and pleuritic chest pain) previously have been used as outcome variables in pneumonia studies, ${ }^{25}$ and COVID-19 convalescents report long-term breathlessness, chest pain and fatigue. ${ }^{26}$ Based on anecdotal evidence, a single course of COVID-19 revealed changes in CAT score from $\mathrm{CAT}=5$ prior to onset of symptoms to a peak of CAT $=31$ and a CAT $=14$ after a total of 40 days (A. Mollerup, 2020).

Although the change in CAT score from baseline to follow-up at day 30 is the primary outcome, the CAT score as repeated daily measurements throughout the active intervention period of 30 days is supposed to contribute to a more thorough understanding of how the individual symptoms may intercorrelate and at what point a potential effect of the PEP flute intervention may initiate and peak.

\section{Secondary outcomes}

The secondary outcomes are comparison between the intervention group and the usual care group of the number of hospital admissions and use of antibiotics during the follow-up period. Presence of COVID-19 symptoms on days 30,90 and 180 and the CAT at days 90 and 180 will be assessed. Moreover, the number of participants with serious adverse events (SAEs) during the 30-day intervention period will be evaluated. Finally, potential subgroup effects by sex, age, comorbidity and Body Mass Index at study entry will be explored for all outcomes as various conditions and comorbidities such as diabetes, hypertension and other chronic diseases have been pointed out as prognostic risk factors. ${ }^{5}$ Registry data of diagnosed comorbidity will also be valuable in the interpretation of symptoms like dyspnoea and chest tightness, which may be overlaps between an underlying disease like heart failure and the present course of COVID-19.

\section{Statistical plan and data analysis}

Both intention-to-treat (ITT) and per-protocol analyses will be performed. The ITT population consists of all randomised participants irrespective of whether the participant received study intervention or whether the participant complied to the study protocol in the treatment group to which the participant was assigned at randomisation. The per-protocol population is defined as participants with a baseline measure of primary outcome and a follow-up measure of primary outcome at the primary assessment call (day 30). As regards the intervention group, participants fulfil the per-protocol criteria if they have complied to the PEP flute intervention for as long as respiratory symptoms are still reported in the CAT score. These data are accessible through the daily self-reports. Participants in the usual care group fulfil the per-protocol criteria if they have no major protocol violations; that is, they have not reported the use of a PEP flute or treatment related to the respiratory system from a physiotherapist.

A statistical analysis plan that describes the details of the planned statistical analyses will be produced before the last patient's last visit, that is, 30-day follow-up. Assessments of changes from baseline and construction of CIs for continuous measures will be based on analysis of covariance, including group as the main factor and baseline measure of outcome as covariate. Superiority will be claimed if the computed $95 \% \mathrm{CI}$ of the estimated group difference in primary outcome does not include 0 in the ITT population. All statistical tests will be two-sided and statistical significance will be claimed if the computed $\mathrm{p}$ value is $<0.05$.

Interactions between intervention status and baseline participant groupings, that is, sex and age, will be prioritised as a priori subgroup analyses for the primary and secondary outcomes.

Determination of sample size according to the primary outcome, that is, the self-reported symptom CAT score, was based on reported symptom scores in a previous study of community-acquired pneumonia. ${ }^{25}$ On a $0-100$ value scale (higher values indicate more symptoms), the mean symptom score at the time of diagnosis was 51.7 (SD 20.1). We used these scores as reference. Hence, we assumed the mean CAT score in the PEP-CoV-trial at baseline to be 20.0 (SD 10.0). A minimal clinical reported difference (MCID) of 2.0 on the CAT scale has been reported from clinical studies of COPD rehabilitation. ${ }^{27}$ Based on this MCID, the assumed mean CAT score at baseline, a significance level of $5 \%$ and a power of 0.8 , we have estimated a need of including $n>141$ in each group. With consideration of potential dropouts in a heterogenous sample, we assess that inclusion of 200 participants in each intervention arm will be an adequate number. A mitigation strategy has been developed to be executed in case of recruitment problems. An interim analysis showed that the mean CAT=12.8 (SD 6.5) at baseline after recruiting 109 participants. No other interim analyses are planned. At present, the prevalence of hospital admission in Denmark is approximately $6 \%$ and, as such, we should expect 30 participants being hospitalised during the active intervention period of 30 days. However, we have not estimated sample size based on hospital admission as outcome variable.

\section{ETHICS AND DISSEMINATION}

The use of PEP flute is considered a low-risk intervention with no expected side effect. Since the interventions will be delivered in combination with standard treatment and we will be closely monitoring potential side effects, we anticipate no ethical issues. The intervention is considered justifiable in a health research ethics perspective. Ethical approval has been granted by the local health ethics committee (journal number H-20035929). The Danish Data Protection Agency has approved conduct of the trial (Capital Region: P-2020-879). An inquiry about the study has been directed to the Danish Medicines Agency because the PEP flute is classified as a medical device. No approval from the Agency is needed since the flute is used for a purpose within the CE classification 
(Agency reference number: 2020051572). It has not been a requirement to compose a data monitoring committee. The trial is exploratory with a design that needs to adapt according to how the pandemic develops and the governmental countermeasures, for example, as regards testing and restrictions. The trial is internally monitored, evaluated and adjusted accordingly.

Prior to screening, all potential trial participants are informed, both orally and in writing, about the purpose of this trial, its process and potential risks, as well as costs and benefits of participation. After the information is delivered, read and understood, voluntary informed consent is given by the participant by signing an e-consent form before trial participation can take place.

Protocol deviations and AEs are recorded by the study staff (AM and ASB). The principal investigator and project manager (AM) monitors and does follow-up of possible AEs and SAEs throughout the study. These procedures are qualified by use of templates from the Danish good clinical practice (GCP) units. $^{28}$

\section{POTENTIAL OUTCOMES AND IMPACT}

The PEP-CoV project is an innovative project niched between screening/prevention through vaccine and hospitalisation/critical illness treatment. This is an important area that, to the best of our knowledge, has received limited attention from both research and health authorities. Coronavirus will continue to be present for the next several years. Thus, many people will become infected by the virus and develop COVID-19, and as a worldwide response to the pandemic, we need to focus on self-care. The PEP-CoV trial aims to prevent serious lung disease and possibly shorten the course of the disease with the use of a simple, cheap and accessible intervention, a PEP flute.

It is difficult to estimate how many hospitalisations among the group of people having COVID-19 could have been avoided by the individual's use of a PEP flute. However, a PEP flute including postal deliverance costs approximately $€ 10$; a regular hospital bed costs around $€ 1000$ a day, whereas an ICU bed usually costs $€ 2000$ $€ 5000$ a day. The PEP flute self-care intervention is feasible and easy to use. If it proves to be effective, it will be easy to implement as a public health intervention. This may result in less sick leaves and less strain for the individual and the family. Moreover, potentially less severe courses of COVID-19 will reduce the overall burden of the healthcare system and the society, whereby we can ensure continued normal high activity in the healthcare system. Handling the PEP flute as a self-care tool during quarantine in one's own home may contribute to a sense of mastery and coping to potentially impact the course of disease through self-care. These latter perspectives may be explored subsequently in a qualitative study design.

According to the ethical approval, the trial is obliged to recruit by a single invitation letter only, sent to the individual's official e-Boks and then await a request for further contact from the eligible participant. Many people check their e-Boks only occasionally. Other eligible participants may feel too sick to overcome this task. Hence, a large sample of individuals tested positive for SARS-CoV-2 will be invited to the trial with only very few to ask for contact. This may challenge a non-selective recruitment although the inclusion criteria are fewer than in many other RCTs.

Although warranted, it is not possible to deliver a placebo PEP flute intervention. Thus, blinding of the patients and treatment providers is not feasible. Because COVID-19 is a novel disease, this study is explorative in relation to using self-reported measurements from COPD treatment, that is, the CAT score as the primary outcome variable. In the design process, it was considered to add objective measures like oxygen saturation, body temperature and/or infectious biomarkers as outcome variables. However, the quarantine restrictions made this choice not feasible and the subsequent implementation of potential positive findings in a public health context advocated for the opt-out of objective measures. However, these issues of both the CAT as outcome measure and the lack of objective measures call for attention in the later discussion of the results of the trial.

There is a risk of contamination across arms as participants can acquire the PEP flute as over-the-counter medical equipment. The participants are asked at follow-up if they have used a PEP flute and/or have received any physiotherapeutic treatment. As data will be analysed both as regards ITT and per protocol, this will be directed in the interpretation of the results.

\section{TRIAL STATUS}

At submission of this article, recruitment to the trial is ongoing with a total of 375 participants enrolled. The protocol was first prospectively registered (www.ClinicalTrials.gov, NCT04530435) on 27 August 2020. No amendments have been made to the protocol (V.3.0, 14 July 2020) since recruitment of the first participant. Minor amendments have been made to the registration on 16 December 2020, with clarification of outcome measurements (general and respiratory symptoms). Recruitment was started on 6 October 2020, and the first participant was enrolled on this date as well. Data of test-positive individuals are provided from the aforementioned four microbiological departments. Recruitment was initiated based on data from only one of the departments in the Capital Region to ensure feasibility of the data management process. One by one, the other departments were enrolled and since the end of October, we have obtained data of all individuals with tests analysed by the regional microbiological departments of the two regions.

\section{Author affiliations}

${ }^{1}$ Research Unit for Dietary Studies, The Parker Institute, Frederiksberg, Copenhagen University Hospital, Frederiksberg, Denmark

${ }^{2}$ The Bachelor of Science in Nursing Programme, The Danish Deaconess

Community Frederiksberg, University College Copenhagen, Copenhagen, Denmark 
${ }^{3}$ The Parker Institute, Frederiksberg, Copenhagen University Hospital, Frederiksberg, Denmark

${ }^{4}$ Department of Clinical Medicine, Faculty of Health and Medical Sciences,

University of Copenhagen, Copenhagen, Denmark

${ }^{5}$ Department of Neurology, Copenhagen University Hospital - Bispebjerg, Copenhagen, Denmark

${ }^{6}$ Department of Infectious Diseases, Copenhagen University Hospital - Hvidovre, Copenhagen, Denmark

${ }^{7}$ Department of Physio- and Occupational Therapy and PMR-C, Copenhagen University Hospital - Hvidovre, Copenhagen, Denmark

${ }^{8}$ Section for General Practice, Department of Public Health, University of Copenhagen, Copenhagen, Denmark

Acknowledgements By sharing their experiences of disease course, the COVID-19 convalescents are both acknowledged for valuable contributions to the trial design.

Contributors AM forwarded the idea and MKS gathered the project team. AM, MKS, SCL, MH, LMK, NW and BLH all contributed to the study conception and design. AM is the project manager. LMK steered the production of instruction videos. ASB steered the set-up of data management system supervised by MH. AM and ASB both handle recruitment, data collection, deliverance of intervention and assessment of all participants. AM handles the hotline and request of advice from participants including any adverse events. MH developed the allocation sequence. SCL manages data and statistical analysis. NW has the medical responsibility in the conduct of the trial and BLH is project lead. AM drafted the manuscript and all authors commented on previous versions of the manuscript. All authors read and approved the final manuscript, and will contribute to the subsequent publication of findings according to the guidelines set forth by the International Committee of Medical Journal Editors. No professional writers will be part of the reporting and publication of the results from the PEP-CoV trial.

Funding This work was supported by Innovation Fund Denmark (grant number 0211-00023B) and a grant (unnumbered) from the Danish Nursing Council. The Parker Institute, Bispebjerg and Frederiksberg Hospital is supported by a core grant from the Oak Foundation.

Disclaimer The funders have no role in the design, conduct, collection of data, analysis, writing or reporting of the trial.

Competing interests None declared.

Patient and public involvement Patients and/or the public were not involved in the design, or conduct, or reporting or dissemination plans of this research.

Patient consent for publication Not required.

Provenance and peer review Not commissioned; externally peer reviewed.

Open access This is an open access article distributed in accordance with the Creative Commons Attribution Non Commercial (CC BY-NC 4.0) license, which permits others to distribute, remix, adapt, build upon this work non-commercially, and license their derivative works on different terms, provided the original work is properly cited, appropriate credit is given, any changes made indicated, and the use is non-commercial. See: http://creativecommons.org/licenses/by-nc/4.0/.

\section{ORCID iDs}

Annette Mollerup http://orcid.org/0000-0002-8161-2214

Nina Weis http://orcid.org/0000-0002-3133-2724

Berit Lilienthal Heitmann http://orcid.org/0000-0002-6809-4504

\section{REFERENCES}

1 Phua J, Weng L, Ling L, et al. Intensive care management of coronavirus disease 2019 (COVID-19): challenges and recommendations. Lancet Respir Med 2020;8:506-17.

2 Reilev M, Kristensen KB, Pottegård A. Characteristics and predictors of hospitalization and death in the first 9,519 cases with a positive RT-PCR test for SARS-CoV-2 in Denmark: a nationwide cohort. medRxiv 2020.

3 Haase N, Plovsing R, Christensen S, et al. Characteristics, interventions, and longer term outcomes of COVID-19 ICU patients in Denmark-A nationwide, observational study. Acta Anaesthesiol Scand 2021;65:68-75.
4 WHO. Covid-19: icd-10 coding guidance 1 18, 2020. Available: https://www.who.int/classifications/icd/COVID-19-coding-icd10.pdf [Accessed 12 Feb 2021].

5 Docherty AB, Harrison EM, Green CA. Features of 20133 UK patients in hospital with covid-19 using the ISARIC WHO Clinical Characterisation Protocol: prospective observational cohort study. BMJ Br Med journal 2020;369:m1985.

6 Beigel JH, Tomashek KM, Dodd LE, et al. Remdesivir for the Treatment of Covid-19 - Final Report. N Engl J Med 2020;383:1813-26.

7 RECOVERY Collaborative Group, Horby P, Lim WS, et al. Dexamethasone in hospitalized patients with Covid-19. N Engl J Med 2021;384:693-704.

8 Kofod LM, Jeschke KN, Krogh-Madsen R. Kontinuerlig CPAP til patienter Kontinuerlig CPAP til patienter Med COVID-19 Med COVID-19. Ugeskr Læger 2020:1-8.

9 Radovanovic D, Rizzi M, Pini S, et al. Helmet CPAP to treat acute hypoxemic respiratory failure in patients with COVID-19: a management strategy proposal. J Clin Med 2020;9:1191.

10 Osadnik CR, McDonald CF, Miller BR, et al. The effect of positive expiratory pressure (PEP) therapy on symptoms, quality of life and incidence of re-exacerbation in patients with acute exacerbations of chronic obstructive pulmonary disease: a multicentre, randomised controlled trial. Thorax 2014;69:137-43.

11 Møller T, Moser C, Adamsen L, et al. Early warning and prevention of pneumonia in acute leukemia by patient education, spirometry, and positive expiratory pressure: a randomized controlled trial. Am J Hematol 2016;91:271-6.

12 Fagevik Olsén M, Lannefors L, Westerdahl E. Positive expiratory pressure - Common clinical applications and physiological effects. Respir Med 2015;109:297-307.

13 O'Neill K, O'Donnell AE, Bradley JM. Airway clearance, mucoactive therapies and pulmonary rehabilitation in bronchiectasis. Respirology 2019;24:227-37.

14 Chan A-W, Tetzlaff JM, Gøtzsche PC, et al. SPIRIT 2013 explanation and elaboration: guidance for protocols of clinical trials. BMJ 2013;346:e7586-42.

15 Sudre $\mathrm{CH}$, Murray B, Varsavsky T. Attributes and predictors of LongCOVID: analysis of COVID cases and their symptoms collected by the Covid symptoms study app. medRxiv 2020.

16 SSI. Covid-19 i Danmark. Epidemiologisk trend og fokus: Symptomer. Copenhagen 2020.

17 Petersen I, Phillips A. Three quarters of people with SARS-CoV-2 infection are asymptomatic: analysis of English household survey data. Clin Epidemiol 2020;12:1039-43.

18 SSI. COVIDmeter. Available: https://covid19.ssi.dk/ overvagningsdata/undersoegelser/covidmeter [Accessed 4 Feb 2021].

19 Harris PA, Taylor R, Thielke R, et al. Research electronic data capture (REDCap)--a metadata-driven methodology and workflow process for providing translational research informatics support. J Biomed Inform 2009;42:377-81.

20 Osadnik CR, McDonald CF, Jones AP, et al. Airway clearance techniques for chronic obstructive pulmonary disease. Cochrane Database Syst Rev 2012:CD008328.

21 Smith JA, Aliverti A, Quaranta M, et al. Chest wall dynamics during voluntary and induced cough in healthy volunteers. J Physiol 2012;590:563-74.

22 Bo A, Friis K, Osborne RH, et al. National indicators of health literacy: ability to understand health information and to engage actively with healthcare providers - a population-based survey among Danish adults. BMC Public Health 2014;14:1-12.

23 Jones PW, Harding G, Berry P, et al. Development and first validation of the COPD assessment test. Eur Respir J 2009;34:648-54.

24 Region Sjælland. Sundhedsfagligt indhold I TeleKol, 2017. Available: https://www.regionsjaelland.dk/Sundhed/samarbejde-og-indsatser/ Telesundhed-KOL/Documents/TeleKOL LANDSDEL SJ/ELLANDS sundhedsfaglige anbefalinger v1.01.pdf

25 Metlay JP, Fine MJ, Schulz R, et al. Measuring symptomatic and functional recovery in patients with community-acquired pneumonia. J Gen Intern Med 1997;12:423-30.

26 Andersen KJ. Åndenød på 8. uge. Sygeplejersken 2020;6:50-1. article in Danish.

27 Jones P, Jenkins C, Agusti A. Healthcare professional user guide. The COPD Assessment Test (CAT), 2018: 1-20. www.catestonline.org

28 GCP-units. The Danish good clinical practice units. Available: https:// gcp-enhed.dk/english/ [Accessed 16 Feb 2021]. 